\title{
Avaliação dos Prontuários Médicos de Hospitais de Ensino do Brasil"
}

\author{
Evaluation of Medical Records in Brazilian \\ Teaching Hospitals
}

Fábia Gama Silva ${ }^{1}$ José Tavares-Neto ${ }^{1}$

PALAVRAS-CHAVE:

- Hospitais Universitários;

- Prontuários;

- Educação Médica.

- Hospitals, University;

- Medical Record;

- Education, Medical.

Recebido em: 07/10/2006

Aprovado em: 07/02/2007

* Apoio: Fundação de Amparo à Pesquisa do Estado da Bahia (Fapesb), CNPq.

${ }^{1}$ Faculdade de Medicina da Bahia, Universidade Federal da Bahia, Bahia, Brasil.

\begin{abstract}
RESUMO
O prontuário do paciente ou do cliente, também denominado prontuário médico, é um elemento fundamental ao bom atendimento e um instrumento de educação permanente e de pesquisa, entre outras finalidades de gerenciamento hospitalar. Neste estudo, foram avaliados os modelos de prontuário utilizados em 77 (73,3\%) dos 105 hospitais filiados à Associação Brasileira de Hospitais Universitários e de Ensino (Abrahue), sendo estudados pela estimativa de escores para vários de seus itens ou de partes referentes à história clínica e à evolução do paciente. O tipo predominante dos prontuários $(92,2 \% ; n=71)$ era em suporte de papel e nenhum no formato eletrônico. Comparados aos prontuários dos hospitais filantrópicos $(n=23)$, os dos públicos (n =54) alcançaram maiores escores ( $p<0,05)$, mas em ambos a totalidade dos itens estudados teve baixa pontuação. Nos itens componentes da anamnese, por exemplo, enquanto o escore máximo esperado era 22, a média foi 4,3 ( \pm 3,7), com limites de 0 e 15 e mediana de 4. Em conclusão, além da reduzida qualidade da maioria dos prontuários estudados, a quase totalidade ainda não incorporou as modernas tecnologias disponibilizadas pela ciência da informação.
\end{abstract}

The customer or patient record, also called medical record, is a fundamental element for providing good patient care, an instrument for permanent education and research, besides serving a variety of hospital management-related purposes. This study appraised the record models used at 77 (73.3\%) of the 105 hospitals belonging to the Brazilian Association of University and Teaching Hospitals (Abrahue) by estimating scores for several of their items or parts of them regarding the clinical history and evolution of the patient. The predominating kind of record (92.2\%; $n=71$ ) was the one written on paper, none was elaborated in electronic format. Comparing the records of public hospitals $(n=54)$ with those of philanthropic hospitals $(n=23)$, the records of the public hospitals reached higher scores ( $p<0.05)$, but in both cases the total of studied items showed low scores. With respect to the items composing the anamnesis for instan$c e$, while the expected maximum score was 22, the mean was $4.3( \pm 3.7)$, with limits of $O$ and 15 and a median of 4. In conclusion, further to the limited quality of most of the studied records, almost none of them had incorporated the modern technologies offered by the information sciences. 


\section{INTRODUÇÃO}

Registrar informações é tarefa e dever diário de todos os profissionais da área de saúde. A reunião dos dados fornecidos pelo paciente, responsáveis legais ou ambos e dos resultados obtidos em qualquer tipo de exame constitui o chamado prontuário médico, também denominado prontuário do paciente ou do cliente, ou mesmo registro médico. Trata-se, portanto, de um documento de extrema relevância que visa, acima de tudo, demonstrar a evolução da pessoa assistida e, subseqüentemente, direcionar o melhor procedimento terapêutico ou de reabilitação, além de assinalar todas as medidas associadas, bem como a ampla variabilidade de cuidados preventivos adotados pelos profissionais de saúde¹.

Isso ficou mais facilitado nas últimas décadas, porque a informatização beneficiou a área médica de forma significativa ao proporcionar a formulação do Prontuário Eletrônico do Paciente (PEP), cujo objetivo é melhorar a qualidade da assistência à saúde. Mas o grau de desenvolvimento tecnológico dos serviços de saúde brasileiros, principalmente na área hospitalar, é muito heterogêneo, e, nesse contexto, o prontuário médico é usualmente um dos últimos a ser informatizado seja por problemas econômico-financeiros, culturais - como resistência à informática por parte dos possíveis usuários -, falta de acesso ao software mais adequado ou até mesmo por supostos impedimentos legais, éticos ou ambos ${ }^{1,2}$.

Apesar disso, no Brasil, os hospitais universitários, de ensino ou escola, fazem parte do nível de maior complexidade do Sistema Único de Saúde e também por essa condição o esperado é que disponham de prontuário do paciente de melhor qualificação. Nesses hospitais, o prontuário - mesmo os modelos em formato tradicional ou suporte em papel - é especialmente útil, porque nessas unidades, além das atividades de assistência, há atividades de ensino e pesquisa. Também, a atuação em ensino é ampla, incluindo cursos de graduação e de pós-graduação das ciências da saúde, a educação permanente e aquela dirigida aos pacientes e à comunidade, bem como o treinamento de pessoal de nível médio e auxiliar ${ }^{3}$.

No entanto, mesmo considerando o atual estágio de desenvolvimento do sistema de saúde do Brasil, se reconhece que a crise nos hospitais de ensino não é apenas financeira e que são necessárias novas políticas públicas para o aprimoramento da gestão hospitalar. Nessa nova realidade, o prontuário mais bem qualificado também serve como instrumento de auditoria e de avaliação das contas médicas ${ }^{4}$. Observandose a tendência de reformulação da educação médica e as novas estratégias pedagógicas que visam ao aumento da eficácia, da eficiência e da efetividade da formação profissional, a implantação do prontuário eletrônico traz vários benefícios ${ }^{5}$.
São pouco conhecidas, entretanto, as características dos prontuários adotados em hospitais universitários, de ensino ou escola do Brasil. Aparentemente, predomina o modelo tradicional, com suporte em papel, e são ainda isolados os casos de instituições brasileiras com prontuários que usam as tecnologias da ciência da informação. Também é desconhecido o conteúdo dos prontuários utilizados nessas instituições de ensino. Assim, o objetivo deste estudo foi realizar a análise secundária de seus dados.

\section{METODOLOGIA}

A abordagem deste estudo é descritiva, do tipo série de casos, abrangendo modelos de prontuários dos hospitais brasileiros que servem como campos de prática aos cursos de graduação em Medicina do Brasil. Foram selecionados todos os 105 Hospitais Universitários, Escola e Auxiliares de Ensino filiados à Associação Brasileira de Hospitais Universitários e Entidades de Ensino (Abrahue); para isso, as direções dos mesmos foram contatadas em até três ocasiões, por meio de correspondência registrada, explicando os objetivos da pesquisa e solicitando o envio dos modelos de prontuário e fichas clínicas. Na primeira correspondência, foi apresentado o projeto de pesquisa.

Neste estudo, foram incluídos todos os modelos de prontuários recebidos entre $1^{\circ}$ de novembro de 2003 e 31 de agosto de 2004, sendo excluídos os prontuários dos hospitais nãorespondentes após 60 dias da terceira solicitação ou que enviaram material impróprio ou incompleto.

Para o registro dos dados, foram criados quatro formulários e, em cada um, assinalados os indicadores pertinentes aos itens próprios aos mesmos; três desses formulários foram destinados à avaliação das fichas clínicas de Medicina Interna do Adulto, Ginecologia-Obstetrícia e Pediatria. No quarto formulário, designado "Prontuário Consolidado", foram computados os dados da folha de evolução médica, folha de enfermagem, folha de anestesia, folha para descrição cirúrgica ou procedimento, resumo de alta, óbito ou transferência. No Quadro 1, estão assinaladas as variáveis computadas em cada formulário e os respectivos escores ou pontuações. Dependendo do item examinado em cada formulário, as pontuações variaram de 0 a 4 (Quadro 1), sendo o valor nulo (0) no caso de a informação não existir ou quando não havia no prontuário espaço para o registro da mesma. 


\section{QUADRO 1}

Variáveis incluídas nos formulários "Medicina Interna do Adulto", "Ginecologia-Obstetrícia”, "Pediatria" e "Formulário consolidado", e seus respectivos escores

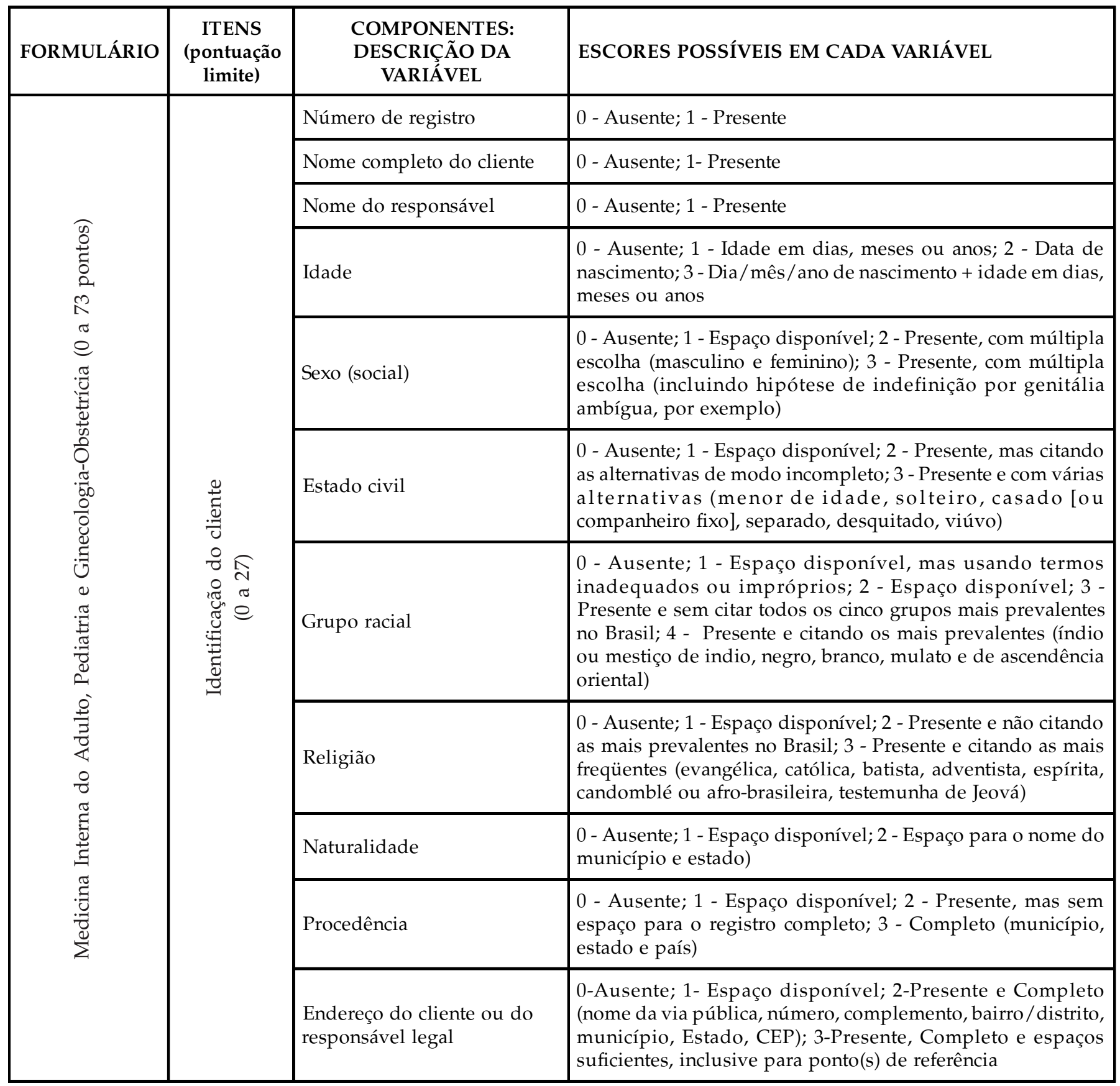




\begin{tabular}{|c|c|c|c|}
\hline FORMULÁRIO & $\begin{array}{l}\text { ITENS } \\
\text { (pontuação } \\
\text { limite) }\end{array}$ & $\begin{array}{l}\text { COMPONENTES: } \\
\text { DESCRIÇÃO DA } \\
\text { VARIÁVEL }\end{array}$ & ESCORES POSSÍVEIS EM CADA VARIÁVEL \\
\hline \multirow{16}{*}{ 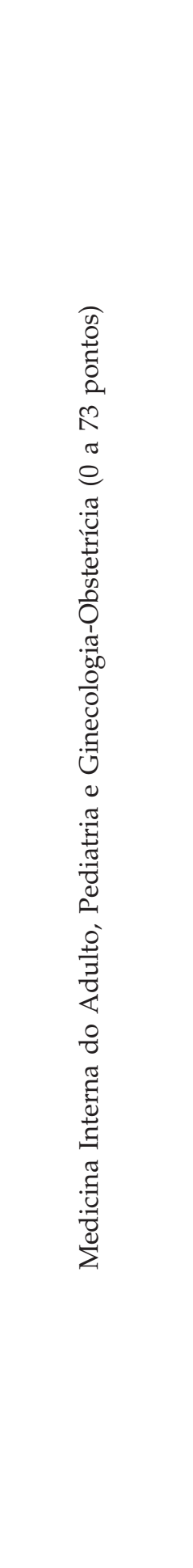 } & \multirow{11}{*}{ 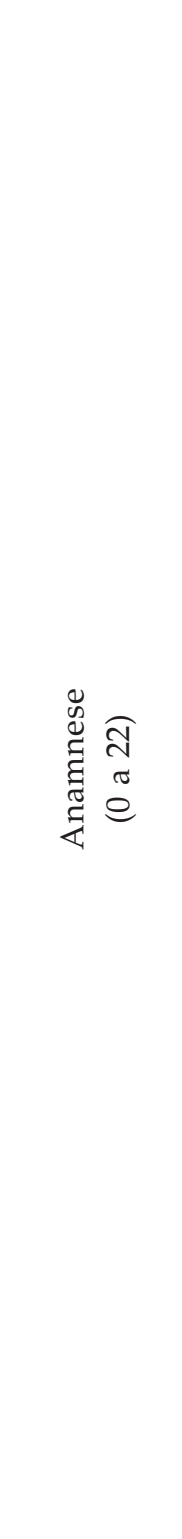 } & Queixa principal & 0 - Ausente; 1 - Presente \\
\hline & & História da doença atual & 0 - Ausente; 1 - Presente \\
\hline & & Revisão de sistemas & $\begin{array}{l}0 \text { - Ausente; } 1 \text { - Espaço disponível; } 2 \text { - Presente, especificando } \\
\text { cada sistema orgânico }\end{array}$ \\
\hline & & $\begin{array}{l}\text { Epidemiologia IST } \\
\text { (Formulário Adulto e GO) }\end{array}$ & $\begin{array}{l}\text { 0-Ausente; } 1 \text { - Espaço disponível; } 2 \text { Presente e citando } \\
\text { algumas alternativas (HIV, HPV, gonorréia, sífilis, etc.) }\end{array}$ \\
\hline & & Imunização (Pediatria) & $\begin{array}{l}0 \text { - Ausente; } 1 \text { - Espaço disponíve } 1 ; 2 \text { - Presente e } \\
\text { especificando alternativas (BCG, pólio, tríplice bacteriana, } \\
\text { hepatite } B \text {, etc.) }\end{array}$ \\
\hline & & Antecedentes de endemias & $\begin{array}{l}0 \text { - Aus e nte ; } 1 \text { - Es p aço disponíve } 1 ; 2 \text { - Presente } \\
\text { (esquistossomose ou doença de Chagas); } 3 \text { - Presente e } \\
\text { citando três ou mais opções (esquistossomose, malária, } \\
\text { doença de Chagas, etc.) }\end{array}$ \\
\hline & & Acidentes ou violências & $\begin{array}{l}0 \text { - Ausente; } 1 \text { - Espaço disponível; } 2 \text { - Presente, mas as } \\
\text { citações são incompletas; } 3 \text { - Presente, com três ou mais } \\
\text { especificações (trânsito, arma de fogo ou branca, queda, } \\
\text { ocupacional, abuso sexual, etc.) }\end{array}$ \\
\hline & & Antecedentes fisiológicos & $\begin{array}{l}0 \text { - Ausente; } 1 \text { - Presente (condição do nascimento, } \\
\text { desenvolvimento na infância, etc.) }\end{array}$ \\
\hline & & $\begin{array}{l}\text { Antecedentes médicos } \\
\text { patológicos }\end{array}$ & $\begin{array}{l}0 \text { - Ausente; } 1 \text { - Espaço disponível; } 2 \text { - Presente, mas sem citar } \\
\text { os mais freqüentes; } 3 \text { - Presente, com campos específicos para } \\
\text { três ou mais (diabetes mellitus, hipertensão arterial sistêmica, } \\
\text { acidente vascular-cerebral, infarto agudo do miocárdio, } \\
\text { hiperlipidemia, hepatite, etc.) }\end{array}$ \\
\hline & & Hábitos de vida & $\begin{array}{l}0 \text { - Ausente; } 1 \text { - Espaço disponível; } 2 \text { - Presente, mas de forma } \\
\text { incompleta; } 3 \text { - Presente e com três ou mais op ções } \\
\text { (tabagismo, alcoolismo, uso de drogas inalatórias, injetáveis, } \\
\text { etc.) }\end{array}$ \\
\hline & & Antecedentes familiares & $\begin{array}{l}0 \text { - Ausente; } 1 \text { - Espaço disponível; } 2 \text { - Presente, mas sem citar } \\
\text { os mais freqüentes; } 3 \text { - Presente, com campos específicos para } \\
\text { três ou mais opções (diabetes mellitus, hipertensão arterial } \\
\text { sistêmica, acidente vascular-cerebral, infarto agudo do } \\
\text { miocárdio, hiperlipidemia, hepatite, etc.) }\end{array}$ \\
\hline & \multirow{5}{*}{ 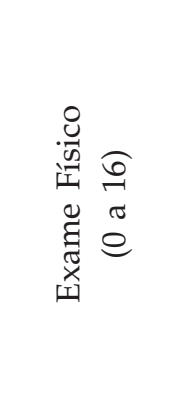 } & Impressão geral & 0 - Ausente; 1 - Presente \\
\hline & & Peso (g ou kg) & 0 - Ausente; 1 - Presente \\
\hline & & Altura (cm ou m) & 0 - Ausente; 1 - Presente \\
\hline & & Índice cintura quadril (ICQ) & $\begin{array}{l}0 \text { - Ausente; } 1 \text { - Ausente, presente apenas circunferência } \\
\text { abdominal; } 2 \text { - Presente apenas o ICQ; } 3 \text { - Presentes ICQ e } \\
\text { circunferência abdominal }\end{array}$ \\
\hline & & Índice de massa corpórea & 0 - Ausente; 1 - Presente \\
\hline
\end{tabular}




\begin{tabular}{|c|c|c|c|}
\hline FORMULÁRIO & $\begin{array}{l}\text { ITENS } \\
\text { (pontuação } \\
\text { limite) }\end{array}$ & $\begin{array}{l}\text { COMPONENTES: } \\
\text { DESCRIÇÃO DA } \\
\text { VARIẤVEL }\end{array}$ & ESCORES POSSÍVEIS EM CADA VARIÁVEL \\
\hline \multirow{14}{*}{ 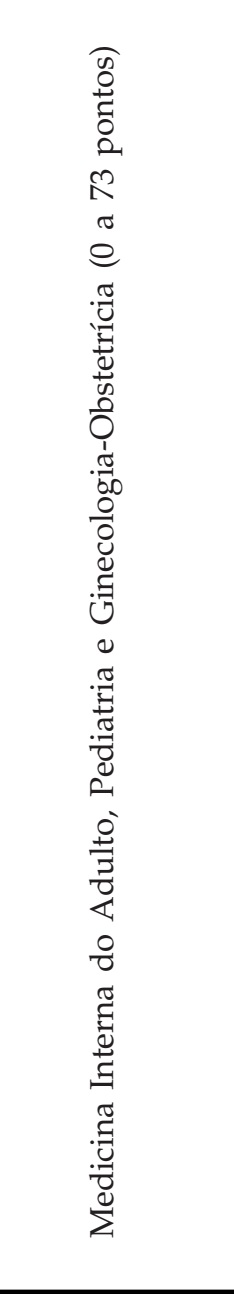 } & \multirow{6}{*}{ 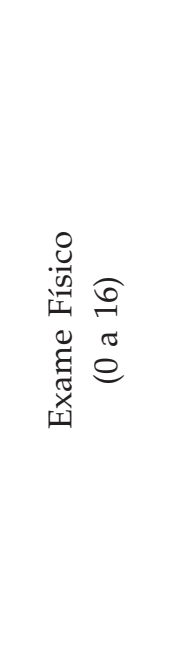 } & Pulso radial (/min.) & 0 - Ausente; 1 - Presente \\
\hline & & $\begin{array}{l}\text { № de movimentos } \\
\text { respiratórios / } \mathrm{min}\end{array}$ & 0 - Ausente; 1 - Presente \\
\hline & & Temperatura ( C) & 0 - Ausente; 1 - Presente \\
\hline & & Tensão arterial (mmHg) & $\begin{array}{l}0 \text { - Ausente; } 1 \text { - Presente incompleto (só uma medida); } 2 \text { - } \\
\text { Presente completo, única medida de cada (pé, sentado e } \\
\text { deitado); } 3 \text { - Presente completo (pé, sentado e deitado), duas } \\
\text { ou mais tomadas de cada }\end{array}$ \\
\hline & & Exame segmentar (subjetivo) & $\begin{array}{l}0 \text { - Ausente; } 1 \text { - Espaço disponível, mas sem indicações do } \\
\text { modelo do exame; } 2 \text { - Presente, com espaços para cada } \\
\text { segmento ou sistema orgânico }\end{array}$ \\
\hline & & Exame segmentar (objetivo) & $\begin{array}{l}0 \text { - Ausente; } 1 \text { - Presente (com as opções para assinalar normal } \\
\text { ou alterado e espaço para descrever apenas as alterações) }\end{array}$ \\
\hline & \multirow{3}{*}{ 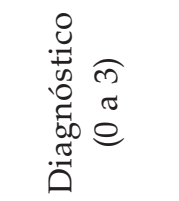 } & Lista de problemas & 0 - Ausente; 1 - Presente \\
\hline & & Hipóteses diagnósticas & 0 - Ausente; 1 - Presente \\
\hline & & Exames diagnósticos & 0 - Ausente; 1 - Presente \\
\hline & \multirow{3}{*}{ 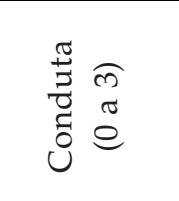 } & Plano terapêutico & 0 - Ausente; 1 - Presente \\
\hline & & Plano educacional & 0 - Ausente; 1 - Presente \\
\hline & & Encaminhamento & 0 - Ausente; 1 - Presente \\
\hline & \multirow{2}{*}{ 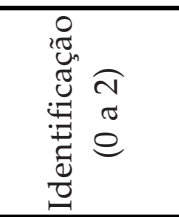 } & $\begin{array}{l}\text { Assinatura do interno ou } \\
\text { médico }\end{array}$ & 0 - Ausente; 1 - Presente \\
\hline & & $\begin{array}{l}\text { Assinatura do médico } \\
\text { responsável }\end{array}$ & 0 - Ausente; 1 - Presente \\
\hline \multirow{7}{*}{ 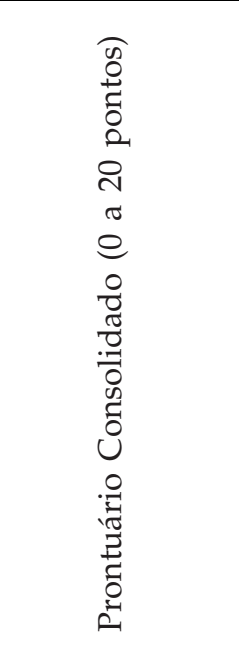 } & \multirow{7}{*}{ 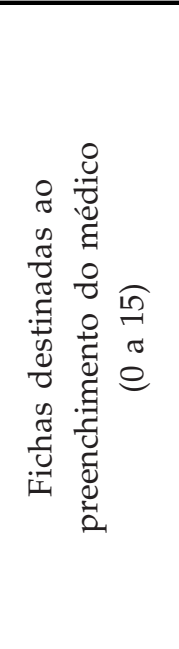 } & Identificação & 0 - Ausente; 1 - Presente \\
\hline & & Anamnese (queixas) & 0 - Ausente; 1 - Presente \\
\hline & & Anamnese (antecedentes) & 0 - Ausente; 1 - Presente \\
\hline & & $\begin{array}{l}\text { Anamnese (história mórbida } \\
\text { pregressa) }\end{array}$ & 0 - Ausente; 1 - Presente \\
\hline & & $\begin{array}{l}\text { Anamnese (história da } \\
\text { doença atual) }\end{array}$ & 0 - Ausente; 1 - Presente \\
\hline & & $\begin{array}{l}\text { Exame físico (cabeça, } \\
\text { pescoço, fâneros, tórax, } \\
\text { abdômen, extremidades, } \\
\text { neurológico, genito-urinário) }\end{array}$ & 0 - Ausente; 1 - Incompleto; 2 - Completo \\
\hline & & Hipótese diagnóstica & 0 - Ausente; 1 - Presente \\
\hline
\end{tabular}




\begin{tabular}{|c|c|c|c|}
\hline FORMULÁRIO & $\begin{array}{l}\text { ITENS } \\
\text { (pontuação } \\
\text { limite) }\end{array}$ & $\begin{array}{l}\text { COMPONENTES: } \\
\text { DESCRIÇÃO DA } \\
\text { VARIÁVEL }\end{array}$ & ESCORES POSSÍVEIS EM CADA VARIÁVEL \\
\hline \multirow{12}{*}{ 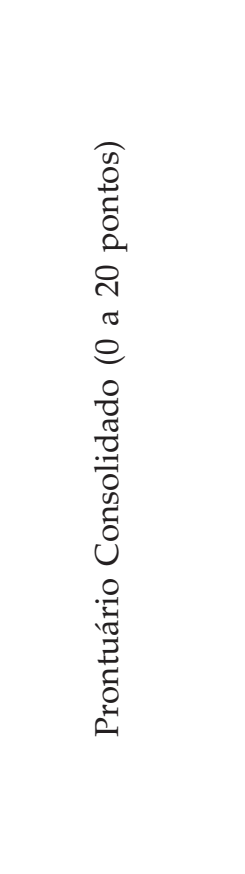 } & \multirow{7}{*}{ 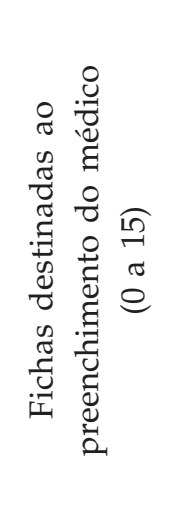 } & Plano terapêutico & 0 - Ausente; 1 - Presente \\
\hline & & Evolução clinica & 0 - Ausente; 1 - Presente \\
\hline & & Pedido de parecer & 0 - Ausente; 1 - Presente \\
\hline & & Prescrição médica & 0 - Ausente; 1 - Presente \\
\hline & & $\begin{array}{l}\text { Resumo de alta, óbito ou } \\
\text { transferência }\end{array}$ & 0 - Ausente; 1 - Presente \\
\hline & & Folha de cirurgia & 0 - Ausente; 1 - Presente \\
\hline & & Folha de anestesia & 0 - Ausente; 1 - Presente \\
\hline & \multirow{5}{*}{ 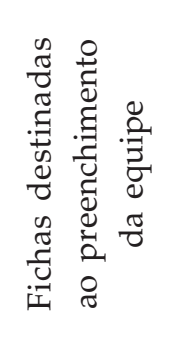 } & Evolução da enfermagem & 0 - Ausente; 1 - Presente \\
\hline & & Fisioterapia & 0 - Ausente; 1 - Presente \\
\hline & & Serviço social & 0 - Ausente; 1 - Presente \\
\hline & & Psicologia & 0 - Ausente; 1 - Presente \\
\hline & & Terapia ocupacional & 0 - Ausente; 1 - Presente \\
\hline
\end{tabular}

Nos formulários "Medicina Interna do Adulto", "Ginecologia-Obstetrícia" e "Pediatria", o escore geral ou total foi composto pelo somatório de seis escores parciais: (a) de identificação da pessoa (número do registro, nome completo, nome do responsável, idade, sexo, estado civil, grupo racial, religião, naturalidade, procedência e endereço), podendo variar de 0 a 27 pontos; (b) sobre a anamnese (queixa principal, história da doença atual, revisão de sistemas, história de infecções sexualmente transmissíveis, ISTs [exclusivamente nos formulários de Medicina Interna do adulto e Ginecologia-Obstetricia], recordatório do calendário de imunização [exclusivamente no formulário de Pediatria], história de acidentes e violências, fatores associados às principais endemias [malária, doença de Chagas, esquistossomose mansônica, entre outras de interesse regional], antecedentes médicos patológicos, fisiológicos e familiares, e hábitos de vida), com limites possíveis de 0 a 22; (c) exame físico (impressão geral, peso, altura, índice cintura quadril, índice de massa corpórea, dados vitais e exame segmentar), com variação possível de 0 a 16; (d) diagnóstico (lista de problemas, hipóteses diagnósticas e campos específicos dos resultados dos exames complementares), de 0 a 3; (e) sobre a conduta (plano terapêutico, educacional e de enca- minhamento), de 0 a 3; (f) identificação do entrevistador (espaço para a assinatura do interno ou do médico-residente e assinatura e carimbo do médico ou médico-supervisor), com variação esperada de 0 a 2.

No formulário do "Prontuário Consolidado", o escore geral corresponde ao somatório de dois escores parciais: (a) fichas destinadas ao preenchimento do médico-assistente (identificação, anamnese, exame físico, hipótese diagnóstica, plano terapêutico, evolução médica, pedido de parecer, prescrição, folha de procedimento cirúrgico, folha da anestesia e resumo de alta, óbito ou transferência), variando de 0 a 15; e (b) fichas da equipe multidisciplinar (relatório da enfermagem, fisioterapia, serviço social, psicologia e terapia ocupacional), de 0 a 5 .

Assim, como acima proposto, os escores máximos correspondem à completitude das informações, ou seja, a presença no prontuário examinado de espaço adequado ao registro da informação e termos ou itens apropriados a cada aspecto examinado.

Adicionalmente, foi também calculado o "escore total" alcançado pelo prontuário examinado, correspondendo ao somatório dos escores parciais registrados nos formulários "Medicina Interna do Adulto", "Ginecologia-Obstetrícia", "Pediatria" e "Prontuário Consolidado". 
Outra variável pesquisada foi o tipo de prontuário (suporte em papel, informatizado ou eletrônico). Também, como variáveis independentes, foram registradas as informações sobre a procedência do hospital (unidade da federação e região geográfica do Brasil) e vinculação administrativa (se pública [federal, estadual ou municipal], filantrópica ou particular).

Na fase de planejamento deste estudo, foram elaborados os quatro formulários de registro dos dados, usandose como indicadores de maior qualidade informações extraídas de livros textos de Semiologia ou Propedêtica Médica $6,7,8$, consulta a professores de Propedêutica e profissionais de Arquivologia, bem como a estudos publicados sobre o tema ${ }^{5,9}$. Posteriormente, os quatro formulários foram submetidos a um estudo-piloto, realizado a partir do exame de dez prontuários, escolhidos aleatoriamente, entre os 77 recebidos, e por três observadores independentes. Na etapa final do estudo-piloto, em reuniões conjuntas com os três observadores e o docente responsável, foram mantidos os indicadores e escores que alcançaram consenso, e revisados um a um os itens conflitantes, até se chegar à proposição consensual. Só após isso, foi iniciada a análise dos 77 prontuários.

Os dados obtidos foram transcritos para um banco de dados criado no Statistical Package for Social Science (SPSS for Windows), versão 9.0. Na análise estatística, cada variável categórica foi descrita pela freqüência e, caso fosse indicado, estudada pelo teste do qui-quadrado. As variáveis quantitativas sem distribuição normal ou do tipo discreta foram descritas pela média ( \pm desvio-padrão) e mediana, e analisadas pelo teste de Mann-Whitney, comparando os respectivos ranks médios. Nesses testes estatísticos, o resultado foi considerado significante quando a probabilidade $(\mathrm{p})$ da ocorrência de erro do tipo I (alfa) foi d" 5\% (ou p $\left.d^{\prime \prime} 0,05\right)$.

\section{RESULTADOS}

Dos 105 hospitais filiados à Abrahue, 26,7\% $(n=28)$ não enviaram os prontuários. Assim, foram excluídos deste estudo: 26 hospitais $(24,8 \%)$ porque não responderam após a terceira solicitação; e $2(1,9 \%)$ que responderam, porém um deles não enviou o prontuário e o outro enviou o formulário muito incompleto. Portanto, foram estudados os prontuários de 77 (73,3\%) instituições.

Comparando os 28 hospitais excluídos aos 77 respondentes quanto à procedência por região do País (Norte + Nordeste versus Sudeste + Sul + Centro-Oeste), foram observadas, respectivamente, as freqüências de $28,6 \%(n=8)$ e $71,4 \%(n=20)$ vs. $32,5 \%(n=25)$ e $67,5 \%(n=52)$, sendo as mesmas estatisticamente semelhantes $\left(c^{2}=0,14 ; p>0,70\right)$.

Essas 77 instituições hospitalares estão distribuídas em 20 unidades da federação e nas cinco macrorregiões brasileiras, sendo 39\% $(n=30)$ no Sudeste; $27,3 \%(n=21)$ no Nordeste; $24,7 \%(n=19)$ no Sul; $5,2 \%(n=4)$ no Norte; e $3,9 \%(\mathrm{n}=3)$ no Centro-Oeste. O maior número de prontuários (53,3\%; $n=41)$ foi procedente de hospitais dos estados de São Paulo $(15,6 \% ; n=12)$, Rio de Janeiro $(14,3 \% ; n=$ 11), Paraná $(11,7 \% ; n=9)$ e Rio Grande do Sul $(11,7 \% ; n=9)$.

Quanto à esfera gestora dos hospitais pesquisados, a maioria $(n=54)$ era pública: $41,6 \%(n=32)$ federais; $24,7 \%$ $(n=19)$ estaduais; e 3,9\% $(n=3)$ municipais. Os hospitais filantrópicos representaram 29,9\% $(n=23)$ do total. Nenhum era particular.

Quanto ao tipo de prontuário, nenhum dos 77 estudados foi eletrônico, e só $7,8 \%(n=6)$ usavam algum recurso de informatização. Portanto, a maioria $(92,2 \% ; n=71)$ dos prontuários era do tipo convencional (suporte em papel).

Como os 77 hospitais respondentes têm clientela (geral, adulta, materna, pediátrica ou materno-infantil) ou atividades (gerais, clínicas, clínico-cirúrgicas ou obstétricas) muito variáveis, só foi possível o registro de 58 formulários de Medicina Interna do Adulto, 23 de Ginecologia-Obstetrícia e 23 de Pediatria. De todos os prontuários estudados ( $\mathrm{n}=77)$, foi possível gerar o "formulário consolidado".

Na Tabela 1, foi registrada a distribuição dos escores totais observados em cada um dos formulários e o tipo de vinculação administrativa da instituição (pública vs. filantrópica). Nos formulários Medicina Interna do Adulto, Pediatria e Ginecologia-Obstetrícia, a pontuação máxima esperada, em cada um, era de 73 pontos, mas os máximos observados foram 46, 45 e 39, respectivamente; os valores das medianas corresponderam, no geral, a menos de $42 \%$ do total esperado. Nesses três formulários, apenas o de Medicina Interna do Adulto alcançou maior pontuação ( $\mathrm{p}<$ $0,03)$ para as entidades públicas, tendo os demais formulários resultados semelhantes $(p>0,69)$ em ambos os tipos de instituições. Apesar disso, foi baixo o valor da mediana (= 24) observado no formulário de Medicina Interna do Adulto das entidades públicas, ou menos de $33 \%$ do total esperado (=73).

As pontuações do "Prontuário Consolidado" das instituições públicas e filantrópicas foram semelhantes $(>0,75)$, e o valor máximo observado (um caso com 17 pontos) correspondeu a $85 \%$ do máximo esperado $(n=20)$, e a mediana $(=11)$ a $55 \%$. 
TABELA 1

Escores totais registrados nos quatro formulários e segundo o tipo de administração da instituição, distribuídos conforme seus limites (mínimo e máximo), mediana (Md) e média e desvio-padrão (M \pm DP)

\begin{tabular}{|c|c|c|c|c|c|c|c|c|c|c|}
\hline \multirow{3}{*}{$\begin{array}{c}\text { Formulário } \\
\text { (número de casos) }\end{array}$} & & & & \multicolumn{6}{|c|}{ Tipo de Administração } & \multirow[b]{3}{*}{$\mathrm{p}^{*}$} \\
\hline & \multicolumn{3}{|c|}{ Escore total } & \multicolumn{3}{|c|}{ Pública } & \multicolumn{3}{|c|}{ Filantrópica } & \\
\hline & Limites & Md & $\mathrm{M} \pm \mathrm{DP}$ & Limites & Md & $\mathrm{M} \pm \mathrm{DP}$ & Limites & Md & $\mathrm{M} \pm \mathrm{DP}$ & \\
\hline $\begin{array}{l}\text { Medicina Interna do } \\
\text { Adulto }(n=58)\end{array}$ & $(2-46)$ & 22 & $23,0(10,3)$ & $(3-46)$ & 24 & $25,0(10,2)$ & $(2-32)$ & 20,0 & $18,5(9,3)$ & $<0,03$ \\
\hline Pediatria $(n=23)$ & $(14-45)$ & 28 & $28,8(6,5)$ & $(14-45)$ & 28 & $28,7(7,2)$ & $(26-33)$ & 29 & $29(3,0)$ & $>0,69$ \\
\hline $\begin{array}{l}\text { Ginecologia-Obstetrícia } \\
(\mathrm{n}=23)\end{array}$ & $(11-39)$ & 30 & $27,1(8,9)$ & $(11-39)$ & 30 & $27,1(8,4)$ & $(16-39)$ & 27 & $27,3(12,4)$ & $>0,82$ \\
\hline $\begin{array}{l}\text { "Prontuário Consolidado" } \\
(\mathrm{n}=77)\end{array}$ & $(4-17)$ & 11 & $10,9(3,1)$ & $(5-17)$ & 11 & $11,1(2,9)$ & $(4-15)$ & 11 & $10,6(3,9)$ & $>0,75$ \\
\hline
\end{tabular}

${ }^{*}$ ) Teste de Mann-Whitney.

Como mostra a Tabela 2, segundo a procedência geográfica dos hospitais (regiões Norte e Nordeste versus Sul mais Sudeste e Centro-Oeste), não foram observadas pontuações estatisticamente diferentes $(p>0,34)$ entre os escores registrados nos quatro formulários pesquisados.

TABELA 2

Distribuição dos escores totais registrados nos quatro formulários, distribuídos conforme seus limites (mínimo e máximo), mediana (Md) e média e desvio-padrão $(\mathrm{M} \pm \mathrm{DP})$, conforme a localização geográfica da instituição

\begin{tabular}{|c|c|c|c|c|c|c|c|c|c|c|}
\hline \multirow{3}{*}{$\begin{array}{c}\text { Formulário } \\
\text { (número de casos) }\end{array}$} & \multicolumn{9}{|c|}{ Região Geográfica } & \multirow[b]{3}{*}{$\mathrm{p}^{*}$} \\
\hline & \multicolumn{3}{|c|}{ Escore total } & \multicolumn{3}{|c|}{ Norte + Nordeste } & \multicolumn{3}{|c|}{$\begin{array}{l}\text { Sul + Sudeste + } \\
\text { Centro-Oeste }\end{array}$} & \\
\hline & Limites & Md & $\mathrm{M} \pm \mathrm{DP}$ & Limites & Md & $\mathrm{M} \pm \mathrm{DP}$ & Limites & Md & $\mathrm{M} \pm \mathrm{DP}$ & \\
\hline $\begin{array}{l}\text { Medicina Interna do } \\
\text { Adulto }(n=58)\end{array}$ & $(2-46)$ & 22 & $23,0(10,3)$ & $(2-45)$ & 23,0 & $24,4(10,8)$ & $(3-46)$ & 22 & $22,4(10,1)$ & $>0,50$ \\
\hline Pediatria $(n=23)$ & $(14-45)$ & 28 & $28,8(6,5)$ & $(20-37)$ & 31 & $30,3(5,9)$ & $(14-45)$ & 27,5 & $28,2(6,7)$ & $>0,35$ \\
\hline $\begin{array}{l}\text { Ginecologia-Obstetrícia } \\
(\mathrm{n}=23)\end{array}$ & $(11-39)$ & 30 & $27,1(8,9)$ & $(13-37)$ & 30 & $27,3(8,6)$ & $(11-39)$ & 29 & $27,1(8,9)$ & $>0,92$ \\
\hline $\begin{array}{l}\text { "Prontuário Consolidado" } \\
(\mathrm{n}=77)\end{array}$ & $(4-17)$ & 11 & $10,9(3,1)$ & $(5-16)$ & 12 & $11,5(3,1)$ & $(4-17)$ & 11 & $10,8(3,0)$ & $>0,34$ \\
\hline
\end{tabular}

(*) Teste de Mann-Whitney. 
Considerando os valores totais dos escores parciais observados nos formulários "Medicina Interna do Adulto" (= 73), “Ginecologia-Obstetrícia" (=73), "Pediatria" (=73) e "Prontuário Consolidado" (=20), o escore total poderia ser de até 239 pontos. No entanto, apenas 8 instituições, todas públicas, tiveram aqueles 4 formulários, e seus escores totais variaram entre 77 e 137 pontos, sendo a mediana de 103,5 e a média de $103,1( \pm 20,5)$.

Por conta desse menor número de hospitais com os quatro formulários, em outro cálculo do escore total só foi incluído o somatório dos escores parciais de dois deles ("Medicina Interna do Adulto" e "Prontuário Consolidado"). Assim, foram computados 58 casos, que apresentaram valores entre 9 e 62 pontos e sendo o máximo possível de 93; nesses casos, a mediana foi 35 e a média $34,2( \pm 12,0)$. Calculado desse modo, o escore total teve mediana e média $( \pm \mathrm{DP})$ nas instituições públicas e filantrópicas, respectivamente, de 36 e 36,4 ( $\pm 11,8)$ vs. 31,5 e $29,3( \pm 10,7)$, mas essa diferença ficou próxima ao limite de significância estatística (teste de Mann-Whitney: $Z=-1,859 ; p=0,063$ ).

Devido ao maior número de casos $(\mathrm{n}=58)$, os escores dos componentes do formulário "Medicina Interna do Adulto" (identificação do paciente, anamnese, exame físico, diagnóstico, conduta e identificação do médico) foram comparados entre aqueles dos prontuários dos hospitais filantrópicos vs. públicos (Tabela 3). Exceto quanto aos dados de anamnese ( $p>$ $0,35)$, exame físico ( $p>0,53)$ e identificação do médico $(p>0,59)$, os demais itens alcançaram valores significativamente mais elevados $(\mathrm{p}<0,02)$ nos formulários extraídos de prontuários dos hospitais públicos. Porém, no geral, os 58 casos no item anamnese tiveram valores com limites entre 0 e 15 pontos (máximo possível de até 22$)$, mediana de 4 e média de $4,3( \pm 3,7)$.

TABELA 3

Análise das médias dos escores parciais dos itens componentes do formulário de Medicina Interna do Adulto, por tipo de administração hospitalar

\begin{tabular}{l|c|c|c|c|c|c|c}
\hline \multirow{2}{*}{ Tipo de Administração } & \multicolumn{7}{|c}{ Média $\left( \pm\right.$ desvio-padrão) $^{\prime}$} \\
\cline { 2 - 8 } & $\mathbf{n}$ & $\mathbf{I d C}^{\mathrm{a}}$ & Anam $^{\mathrm{b}}$ & Exf $^{\mathrm{c}}$ & Diagd $^{\mathrm{d}}$ & Cond $^{\mathrm{e}}$ & IdM $^{\mathrm{f}}$ \\
\hline Pública & 40 & $11,9( \pm 5,3)$ & $4,6( \pm 3,8)$ & $3,9( \pm 3,7)$ & $1,6( \pm 0,9)$ & $1,6( \pm 0,9)$ & $1,2( \pm 0,7)$ \\
\hline Filantrópica & 18 & $8,4( \pm 5,2)$ & $3,6( \pm 3,4)$ & $3,8( \pm 3,9)$ & $0,9( \pm 0,8)$ & $0,9( \pm 0,8)$ & $0,8( \pm 0,7)$ \\
\hline $\mathrm{p}^{\mathrm{g}}$ & & $<0,02$ & $>0,35$ & $>0,52$ & $<0,007$ & $<0,007$ & $>0,59$ \\
\hline
\end{tabular}

(a) Identificação do cliente; (b) Anamnese; (c) Exame físico; (d) Diagnóstico; (e) Conduta;

(f) Identificação do médico; (g) Teste de Mann-Whitney.

O Quadro 2 mostra as principais omissões observadas naqueles quatro formulários. Nos itens associados a anamnese, por exemplo, cerca de um quarto $(24,7 \%)$ dos prontuários examinados não tinha espaço para o registro da queixa principal ou do motivo da consulta (ou atendimento); $15,6 \%$ para a história da doença atual; 44,2\% para o registro das queixas relacionadas aos diversos sistemas; e mais da metade dos prontuários não tinha espaço para a história de infecções sexualmente transmissíveis $(67,5 \%)$, recordatório do calendário de imunização $(70,1 \%)$, história de acidentes e violências $(71,4 \%)$, fatores associados às principais endemias (70,1\%) e hábitos de vida $(58,4 \%)$. 


\section{QUADRO 2}

Distribuição das principais deficiências ou ausência de registro nos formulários de "Medicina Interna do Adulto", "Ginecologia-Obstetrícia", "Pediatria" e "Formulário consolidado", extraídos dos prontuários dos hospitais estudados

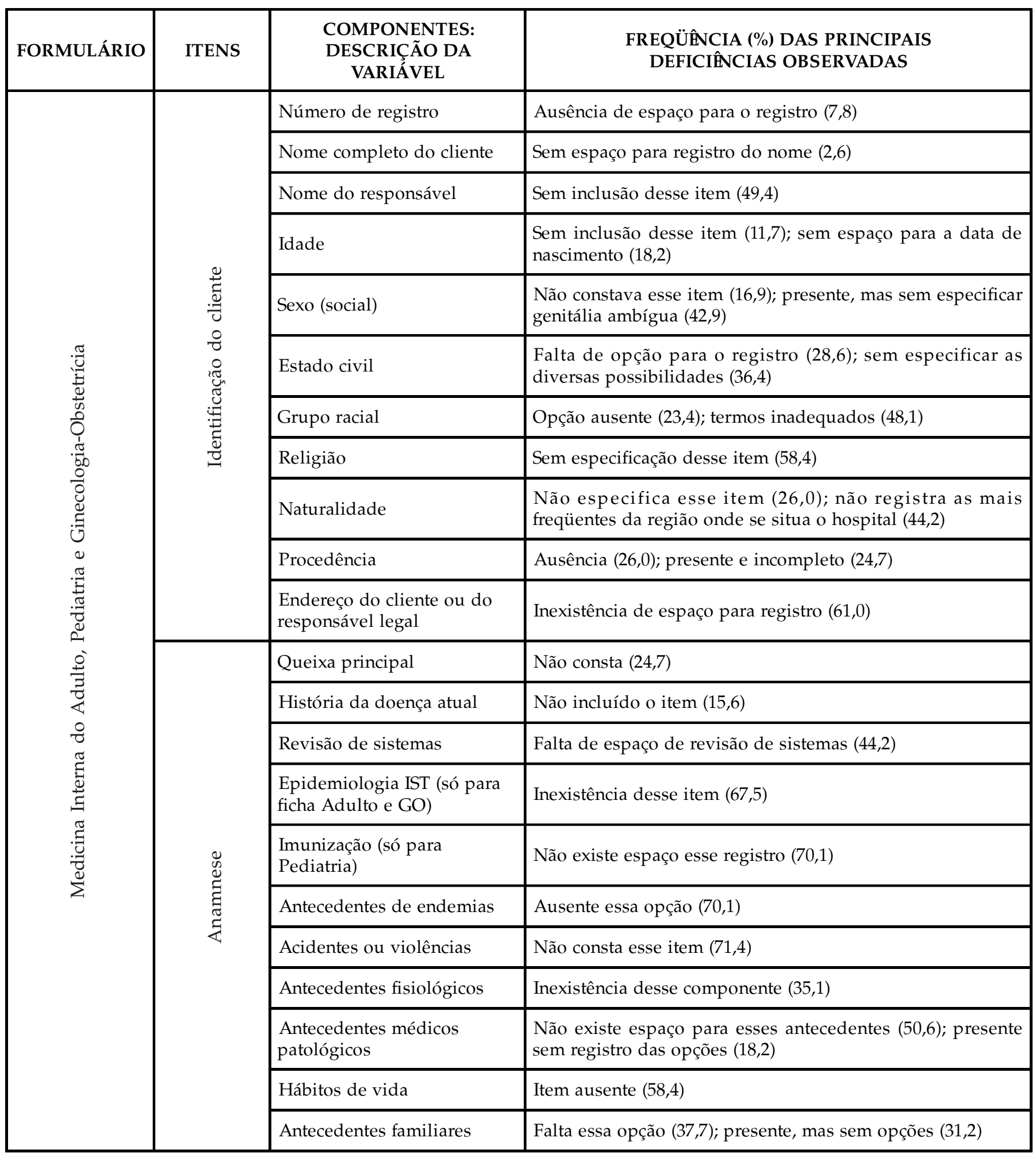




\begin{tabular}{|c|c|c|c|}
\hline FORMULÁRIO & ITENS & $\begin{array}{c}\text { COMPONENTES: } \\
\text { DESCRIÇÃO DA } \\
\text { VARIÂVEL }\end{array}$ & $\begin{array}{l}\text { FREQÜÊNCIA (\%) DAS PRINCIPAIS } \\
\text { DEFICIÊNCIAS OBSERVADAS }\end{array}$ \\
\hline \multirow{10}{*}{ 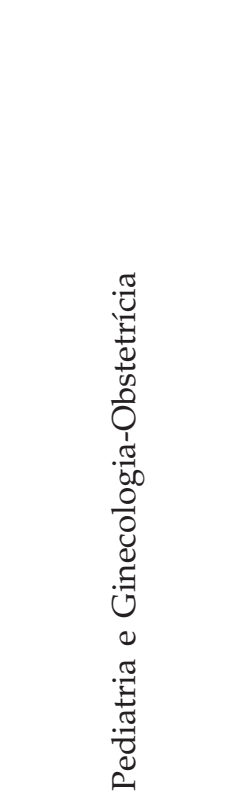 } & \multirow{11}{*}{ 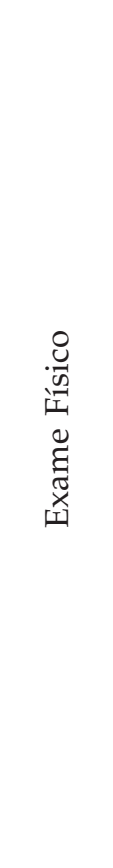 } & Impressão geral & Inexiste espaço para a impressão geral $(49,4)$ \\
\hline & & Peso (g ou kg) & Não consta esse item $(41,6)$ \\
\hline & & Altura $(\mathrm{cm}$ ou m) & Não incluído espaço para esse item $(45,5)$ \\
\hline & & Índice cintura quadril (ICQ) & Falta espaço para ICQ $(74,0)$ \\
\hline & & Índice massa corpórea & Inexistência desse item $(72,7)$ \\
\hline & & Pulso radial (/min.) & Sem espaço para pulso radial $(41,6)$ \\
\hline & & $\begin{array}{l}\text { № de movimentos } \\
\text { respiratórios / min }\end{array}$ & Sem inclusão dessa opção $(45,5)$ \\
\hline & & Temperatura $\left({ }^{\circ} \mathrm{C}\right)$ & Não consta o item $(39,0)$ \\
\hline & & Tensão arterial (mmHg) & $\begin{array}{l}\text { Falta de opção para tensão arterial }(39,0) \text {; presente e } \\
\text { incompleto }(33,8)\end{array}$ \\
\hline & & Exame segmentar (subjetivo) & $\begin{array}{l}\text { Sem inclusão desse item }(31,2) \text {; presente, mas sem sugestão } \\
\text { de modelo do exame }(32,5)\end{array}$ \\
\hline \multirow{9}{*}{ 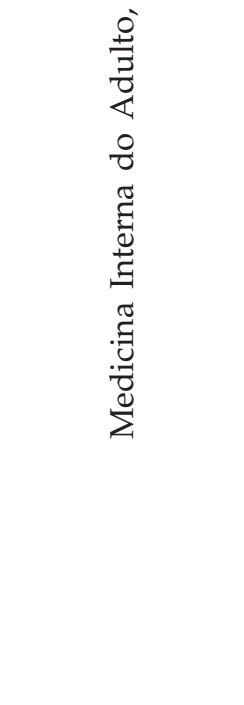 } & & Exame segmentar (objetivo) & Ausência dessa opção $(71,4)$ \\
\hline & \multirow{3}{*}{ 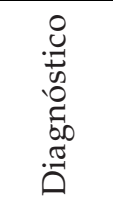 } & Lista de problemas & Falta a opção $(63,6)$ \\
\hline & & Hipóteses diagnósticas & Não consta esse item $(20,8)$ \\
\hline & & Exames complementares & Sem inclusão $(35,1)$ \\
\hline & \multirow{3}{*}{ 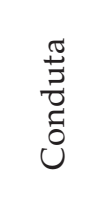 } & Plano terapêutico & Ausência de espaço $(48,1)$ \\
\hline & & Educacional & Falta o item plano educacional $(72,7)$ \\
\hline & & Encaminhamento & Sem espaço para algum tipo de encaminhamento $(50,6)$ \\
\hline & \multirow{2}{*}{ 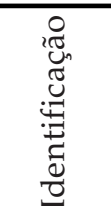 } & $\begin{array}{l}\text { Assinatura do interno ou } \\
\text { médico }\end{array}$ & $\begin{array}{l}\text { Não existe espaço para assinatura do interno e/ou médico } \\
(50,6)\end{array}$ \\
\hline & & $\begin{array}{l}\text { Assinatura do médico } \\
\text { responsável }\end{array}$ & Sem a inclusão desse item $(19,5)$ \\
\hline
\end{tabular}




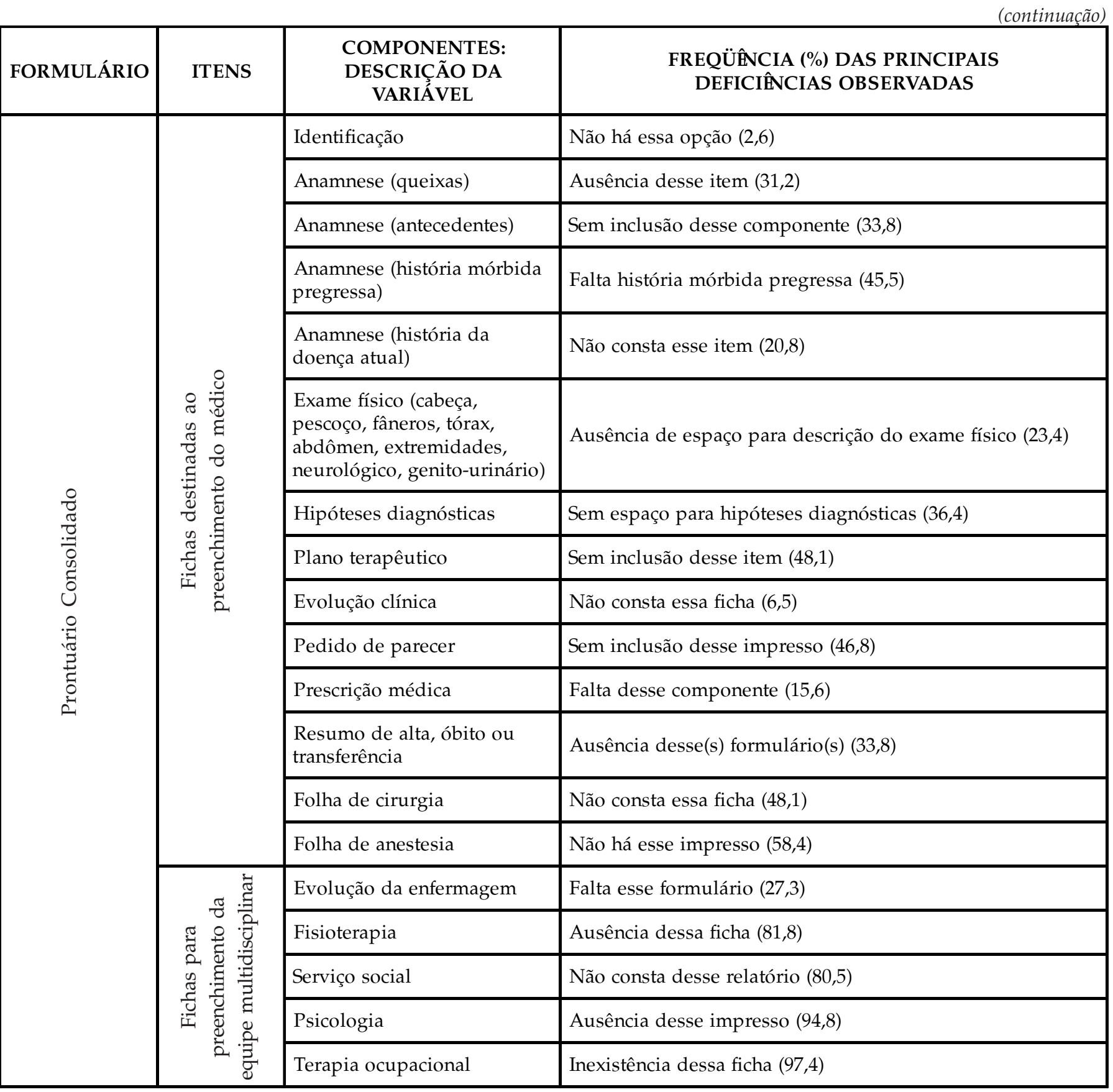

\section{DISCUSSÃO}

A elevada adesão (73,3\%) dos hospitais foi motivada, possivelmente, pela existência de poucos trabalhos sobre esse tema no Brasil e também pelo crescente interesse quanto ao correto registro dos serviços prestados na área da saúde, muitos deles de elevado custo ${ }^{10}$.

Entre os hospitais participantes deste estudo, quase um terço $(29,9 \%)$ é filantrópico, e essa elevada freqüência possi- velmente decorre da forte influência, no Brasil, das associações vinculadas às igrejas cristãs ou porque pertencem a essas entidades religiosas ${ }^{11}$.

Apesar do processo em curso de descentralização promovido especialmente após o advento do Sistema Único de Saúde (SUS), há ainda no Brasil predominância de instituições centralizadas $^{12}$, ratificada pela maior freqüência observada neste estudo de hospitais vinculados ao governo federal 
(41,6\%), especialmente em relação ao baixo percentual $(3,9 \%)$ de hospitais municipais.

Essa tendência administrativa centralizadora do governo federal brasileiro não logrou maior efetividade, considerando-se os muitos relatos do caótico sistema de oferta de serviços de saúde. Apesar disso, o novo modelo preconizado pelo SUS compartilha as responsabilidades com o Ministério da Saúde (esfera federal), as secretarias estaduais de saúde e as prefeituras municipais, sendo este um novo sistema de controle administrativo baseado na descentralização e na municipalização ${ }^{12}$. Assim, com o progresso do novo sistema de saúde, haverá maior proximidade do gestor e dos usuários, e, conseqüentemente, o controle social oferece maiores chances de ser mais eficiente e efetivo.

Contudo, na atual realidade, os custos hospitalares são ainda muito elevados e diretamente responsáveis pela redução de quase $10 \%$ do número de leitos disponíveis ao Sistema Único de Saúde ${ }^{10}$. Em muito colabora para isso ainda não haver no Brasil maior número de unidades de saúde, dos níveis primário e secundário, lastreadas na oferta de serviços e programas de extrema qualidade, até para servirem como modelos multiplicadores e de campos de prática para o treinamento de pessoal. Também, o sucesso do atual sistema de saúde tem como fundamento a consolidação de consistente programa de referência e contra-referência, o qual, por sua vez, é baseado no correto registro de informações pela equipe de saúde de todos os níveis da atenção à saúde. Todavia, o programa de referência e contra-referência dos pacientes tem maior chance de cumprir suas finalidades se estiver fundamentado em prontuário com campos ou itens adequados, ordenados de forma racional, de acordo com o grau de complexidade da unidade de saúde e objetivando a mais completa história do cliente.

Como parte do processo de gestão hospitalar na área assistencial, a oferta de prontuário com maiores possibilidades de registros corretos por parte de seus profissionais, especialmente dos médicos, tem implicações diretas não só nas contas a serem apuradas, mas, fundamentalmente, na qualidade da assistência prestada. No entanto, a análise dos 77 prontuários evidenciou a carência de forma e conteúdo de muitos deles. Como são provenientes de instituições de ensino, teoricamente no ápice do sistema brasileiro de saúde, chega a ser preocupante especular qual será a realidade desses mesmos registros nos outros hospitais terciários brasileiros. Do mesmo modo, é até mais difícil especular qual seria a atual realidade dos formulários ou prontuários utilizados nos serviços de atenção à saúde dos níveis primário ou secundário.

Essa situação preocupante se fundamenta na baixa ou regular qualidade observada na maioria dos prontuários dos hospitais filiados à Abrahue, quando o esperado, pela relevância desse instrumento na área do ensino, era o encontro de valores bem acima da mediana estimada a partir do número total da pontuação máxima calculada. Portanto, em decorrência dos indicadores observados nos prontuários de hospitais da área de ensino, há fundamento para especular que o atual sistema de saúde no Brasil não tem sustentação em base de dados registrada em prontuários ou formulários apropriados aos seus fins.

Exemplo disso foi a pontuação encontrada no item anamnese dos prontuários pesquisados, que muitos autores consideram parte essencial da história clínica ${ }^{6,7,8}$. Mesmo assim, nos 58 formulários estudados, 50\% tiveram pontuação entre 0 e 4 (valor da mediana), e em apenas um hospital o máximo alcançado foi 15 pontos, correspondendo a apenas $68,2 \%$ da pontuação máxima possível, ou 22 pontos.

$\mathrm{O}$ aparente descaso com o mais correto registro das informações de interesse das pessoas assistidas pelo sistema de saúde é, de certo modo, coerente com a freqüência nula de casos de prontuário eletrônico ou mesmo com a baixa freqüência $(7,8 \%)$ daqueles com alguma informatização. Essas observações, especialmente no âmbito dos hospitais universitários brasileiros, também podem ser outro indicador da atual e persistente crise pela qual estes passam nas últimas três décadas, não lhes permitindo acompanhar os recentes avanços da Ciência da Informação ${ }^{4}$.

Mesmo considerando os atuais impedimentos à implantação do prontuário eletrônico ${ }^{2}$ e os resultados observados neste trabalho, os recursos oferecidos pela informática ainda são precariamente utilizados nos hospitais estudados e vinculados à Associação Brasileira de Hospitais Universitários e de Ensino. Essa situação também pode ser outra evidência da falta de recursos e insumos para o desenvolvimento dessas novas tecnologias.

Este estudo também mostra que, antes desse avanço tecnológico, há necessidade de apurada revisão dos diversos itens que compõem o prontuário do cliente, porque as novas tecnologias da informação não suprem as deficiências de registro por falta, exclusivamente, daqueles itens ou de alternativas de registro mais apropriadas.

No Brasil, os hospitais universitários, de ensino ou escola, subordinados ao setor público, são mais antigos, o que explica em parte a presença de prontuários de melhor qualidade entre eles. Mas, em geral, tanto esses como os hospitais filantrópicos têm prontuários de regular ou baixa qualidade. Dessa forma, a revisão e a atualização desses prontuários devem preceder qualquer tentativa de modernização, especialmente se for planejada a informatização ou mudança para o formato eletrônico. 
Também não devem ser negligenciados aspectos regionais ou de interesse de parte da população assistida. Isto porque foi muito freqüente $(70,1 \%)$ em hospitais, em particular naqueles sediados em regiões endêmicas (e.g., esquistossomose mansônica, doença de Chagas, malária, etc.), a falta de qualquer registro ou espaço adequado ao registro de fatores associados a essas endemias. De modo semelhante, a maioria (70,1\%) dos formulários de Pediatria não tinha registros adequados à história de vacinas, em especial daquelas incluídas no Programa Nacional de Imunizações para a faixa etária de 0 a 5 anos.

Em conclusão, a qualidade observada nos prontuários de hospitais da área de ensino, de modo geral, é bastante desalentadora e requer urgentes medidas, inclusive de qualificação de pessoal. Sem mudanças substantivas da maioria dos prontuários examinados, certamente haverá a continuidade dos reflexos negativos sobre as áreas de ensino e pesquisa clínica, entre outras, o que poderá comprometer o desenvolvimento e o aperfeiçoamento do sistema de saúde do Brasil e a assistência da população.

\section{AGRADECIMENTOS}

À Abrahue e às instituições filiadas; e aos acadêmicos de Medicina Daniel Francisco Viriato dos Santos e Ludenor Pereira Lemos-Junior, da Faculdade de Medicina da Bahia da UFBA, pela colaboração na digitação e análise dos resultados e participação no estudo-piloto.

\section{REFERÊNCIAS}

1. Novaes MA. Prontuário Eletrônico do Paciente (PEP). In: I Seminário de Tecnologia da Informação em Saúde; 1998 set. 2-4; Recife, PE.

2. Collazos K, Gotardo RT, Barreto JM. Metodologia para construção do prontuário médico eletrônico. [online]. In: XVIII Congresso Brasileiro de Engenharia Biomédica; 2002; São José dos Campos, SP. [capturado 10 jul. 2003]; Disponível em: http://www.sbis-rj.org/informedica/ebm/ cbeb20 8.pdf.

3. Brasil. Ministério da Educação e Cultura. Departamento de Assuntos Universitários. Comissão do Ensino Médico. Documento $\mathrm{n}^{\circ}$ 2: ensino médico e instituições de saúde. Brasília: MEC; 1974.

4. Brasil.Ministério da Educação e Cultura. Programa de Reestruturação dos Hospitais de Ensino do Ministério da Educação. [online]. Brasília: MEC; 2004. [capturado 14 jul. 2005]. Disponível em: http://www.mec.gov.br.
5. Massad E, Marin HF, Azevedo Neto RS, eds. O prontuário eletrônico do paciente na assistência, informação e conhecimento médico. [online]. São Paulo: H. de F. Marin; 2003. [capturado 14 jul. 2005]; Disponível em: http:// www.netsim.fm.usp.br/dim/livrosdim/prontuario.pdf.

6. Porto CC. Semiologia médica. Rio de Janeiro: GuanabaraKoogan; c2001.

7. Lopez M, Medeiros JL. Semiologia médica: as bases do diagnóstico clínico. Rio de Janeiro: Revinter; 2004.

8. Bates B, Bickley LS, Hoekelman RA. Propedêutica Médica. 7 ed. Rio de Janeiro: Guanabara-Kooga;2001.

9. Costa CGA. Desenvolvimento e avaliação tecnológica de um sistema de prontuário eletrônico do paciente, baseado nos paradigmas da World Wide Web e da engenharia de software. Campinas, SP; 2001. Mestrado [Dissertação] Faculdade de Engenharia Elétrica e da Computação. Universidade Estadual de Campinas.

10. A redução de leitos: criar um código nacional de saúde é vista como uma das necessidades da Saúde. Notícias Hospital. [online]. 2005; 4(46). [capturado 13 maio 2005]; Disponível em: http://www.noticiashospitalares.com.br/ mar2005/htms/capa.htm.

11. Lisboa TC. Breve história dos hospitais: da antigüidade à Idade Contemporânea. Notícias Hospital. 2002; 4(37): 1-30.

12. Pereira LCB. Controle municipal do SUS. [online]. In: Brasil. Ministério da Saúde. DATASUS. Conferencia Nacional de Saúde On-Line. [capturado 10 jun. 2005]; Disponível em: http: / /www.datasus.gov.br/cns/temas/Bresser.htm.

\section{Conflito de Interesse}

Declarou não haver.

\section{Endereço para correspondência}

Fábia Gama Silva

Rua Manoel Gomes de Mendonça, 229 - apto 1501 - Pituba

41810-820 - Salvador - Bahia

e-mail: fabiagama@gmail.com 